\title{
Connexin 40 Promoter-Based Enrichment of Embryonic Stem Cell-Derived Cardiovascular Progenitor Cells
}

\author{
R. David H. Theiss W.-M. Franz
}

Medizinische Klinik und Poliklinik I, Klinikum Grosshadern, Ludwig-Maximilians-Universität, München, Germany

\section{Key Words}

Embryonic stem cells $\cdot$ Connexin $40 \cdot$ Magnetic cell sorting $\cdot$ Cardiovascular progenitor cells $\cdot$ Cardiac tissue engineering

\begin{abstract}
Background: Pluripotent embryonic stem (ES) cells that can differentiate into functional cardiomyocytes as well as vascular cells in cell culture may open the door to cardiovascular cell transplantation. However, the percentage of ES cells in embryoid bodies (EBs) which spontaneously undergo cardiovascular differentiation is low $(<10 \%)$, making strategies for their specific labeling and purification indispensable. Methods: The human connexin 40 (Cx40) promoter was isolated and cloned in the vector pEGFP. The specificity of the construct was initially assessed in Xenopus embryos injected with Cx40-EGFP plasmid DNA. Stable Cx40-EGFP ES cell clones were differentiated and fluorescent cells were enriched manually as well as via fluorescence-activated cell sorting. Characterization of these cells was performed with respect to spontaneous beating as well as via RT-PCRs and immunofluorescent stainings. Results: Cx40-EGFP reporter plasmid injection led to EGFP fluorescence specifically in the abdominal aorta of frog tadpoles. After crude manual enrichment of highly Cx40-EGFP-positive EBs, the appearance
\end{abstract}

R.D. and H.T. contributed equally to this paper. of cardiac and vascular structures was increased approximately 3-fold. Immunofluorescent stainings showed EGFP expression exclusively in vascular-like structures simultaneously expressing von Willebrand factor and in formerly beating areas expressing $\alpha$-actinin. Cx40-EGFP-expressing EBs revealed significantly higher numbers of beating cardiomyocytes and vascular-like structures. Semiquantitative RTPCRs confirmed an enhanced cardiovascular differentiation as shown for the cardiac markers Nkx2.5 and MLC2v, as well as the endothelial marker vascular endothelial cadherin. Conclusions: Our work shows the feasibility of specific labeling and purification of cardiovascular progenitor cells from differentiating EBs based on the $\mathrm{C} \times 40$ promoter. We provide proof of principle that the deleted CD4 ( $\triangle C D 4)$ surface marker-based method for magnetic cell sorting developed by our group will be ideally suitable for transference to this promoter.

Copyright $\odot 2008$ S. Karger AG, Basel
Abbreviations used in this paper

Cx40 connexin 40

EBs embryoid bodies

EGFP enhanced green fluorescent protein

ES embryonic stem

FACS fluorescence-activated cell sorting

MACS magnetic cell sorting

\begin{tabular}{ll}
\hline KARGER & $\oplus$ 2008 S. Karger AG, Basel \\
Fax +4161306 1234 $34-6405 / 08 / 1882-0062 \$ 24.50 / 0$ \\
$\begin{array}{l}\text { E-Mail karger@karger.ch } \\
\text { www.karger.com }\end{array}$ & $\begin{array}{l}\text { Accessible online at: } \\
\text { www.karger.com/cto }\end{array}$
\end{tabular}

Prof. Dr. W.-M. Franz, Medizinische Klinik und Poliklinik I

Ludwig-Maximilians-Universität, Klinikum Grosshadern

Marchioninistrasse 15, DE-81377 München (Germany)

Tel. +49 897095 3094, Fax +49 8970956094

E-Mail wolfgang.franz@med.uni-muenchen.de 


\section{Introduction}

The identification and characterization of embryonic stem (ES) cells that can differentiate into functional cardiomyocytes as well as vascular cells in cell culture may open the door to therapeutic cardiovascular cell transplantation [Vittet et al., 1996; Nir et al., 2003; Gerecht-Nir et al., 2004]. Yet, the percentage of ES cells in embryoid bodies (EBs) which spontaneously undergo cardiovascular differentiation is low $(<10 \%)$ and highly dependent on the ES cell line used as well as on the serum lots available to the researcher. Additionally, ES cell-derived cardiomyocytes terminate their cell cycle followed by formation of multinuclei - a typical feature of terminally differentiated cardiomyocytes [Klug et al., 1996]. Therefore, the proliferative potential of ES-derived cardiomyocytes in vivo is probably limited and the availability of reasonable numbers of cardiomyocytes required to repair a myocardial infarction in men $\left(>10^{8}\right.$ cells) appears to be a major prerequisite yet to be fulfilled for their clinical application [Li et al., 1999]. At the same time, ES cell-derived cardiac tissue will require a sufficient blood supply via newly formed vasculature to warrant its survival in the host myocardium [Hunt, 1998].

Problems yet to be solved for future ES cell-based cardiac therapy comprise (1) reliably high cellular purities to avoid transplantation of undesired cell types leading to teratoma formation and (2) sufficient numbers of cardiovascular cells meant for transplantation. Methods such as fluorescence-activated cell sorting (FACS) or magnetic cell sorting (MACS) allow purification of specific cell types but are dependent on the expression of a specific surface marker that can be recognized by a fluorescent or magnetic microbead-tagged antibody. Yet, for many cell types such as cardiac and vascular cells an appropriate endogenous marker is not known. Therefore, sorting methods have to rely on the introduction of a marker gene under the control of a lineage-specific promoter. In such an effort we have previously labeled and FACS purified ventricular cardiomyocytes expressing enhanced green fluorescent protein (EGFP) [Muller et al., 2000] and subsequently developed a protocol for MACS allowing rapid isolation of highly purified viable ES cell-derived cell types [David et al., 2005].

A novel candidate to transfer our approach to common cardiovascular progenitor cells is the promoter of the connexin 40 ( $\mathrm{Cx} 40)$ gene. In the fetal mammalian heart, $\mathrm{Cx} 40$ is expressed in ventricular and atrial myocytes as well as in the ventricular conduction system [Teunissen and Bierhuizen, 2004]. In addition, endothe- lial cells express Cx40, whereas smooth muscle cells express $\mathrm{Cx} 43$ or $\mathrm{Cx} 45$ [Simon and McWhorter, 2002]. Therefore, the $\mathrm{Cx} 40$ promoter may provide a useful tool to target marker gene expression to cardiovascular derivatives of differentiating ES cells [Seul et al., 1997].

During ES cell differentiation, Cx40 mRNA is expressed at days 3-8 of EB formation [Oyamada et al., 1996]. This background prompted us to perform our analyses at day 6 of differentiation, the earliest possible time point at which EGFP labeling allowed high yields of positive cells representing putative early cardiovascular precursors.

\section{Materials and Methods}

\section{Plasmid Construction}

The human 4.9-kb Cx40 promoter was amplified from human genomic DNA via proof-reading PCR using Pfu polymerase (Stratagene). Primers for cloning of the $\mathrm{Cx} 40$ promoter fragment contained Kpn1 and Age1 restriction sites. Subsequently, the PCR product was inserted into pEGFP (Clontech) after digestion with these 2 enzymes. In detail, the primers were: 5'-GATggtaccGAAAAACACTGGCCGTGTAG-3' (forward primer, Kpn1 site in lower case characters) and $5^{\prime}$-CTATaccggtCTTGGCACAGCCAGGGAAC-3' (backward primer; Agel site in lower case characters). The $\mathrm{C}$ (in bold) in the backward primer corresponds to $-1 \mathrm{bp}$ of the $\mathrm{Cx} 40$ coding region. After sequencing, this vector was used for electroporation of GSES cells and subsequent selection of stable clones.

\section{Xenopus Injections}

Xenopus embryos were injected with $100 \mathrm{pg}$ of Cx40-EGFP plasmid DNA at the 2-cell stage into 1 blastomere according to standard protocols [David et al., 1998]. As injection volume, $5 \mathrm{nl}$ was used. Embryos were obtained by in vitro fertilization, cultivated and injected as described previously [David et al., 1998]. Embryos were kept in $1 \times$ modified Barth's saline solution holding medium until $2-5 \mathrm{~h}$ after injection and then transferred to $0.1 \times$ modified Barth solution holding medium. Staging was performed according to the normal table of Nieuwkoop and Faber. Embryos exhibiting GFP fluorescence were sorted using a Zeiss epifluorescence microscope.

\section{ES Cell Culture and Clone Selection}

Electroporation and isolation of stable clones using the murine ES cell line GSES were performed according to standard protocols with minor modifications [Muller et al., 2000]. Thereby, 5 $\mu \mathrm{g}$ nonlinearized vector was used for electroporation $(240 \mathrm{~V} / 500$ $\mu \mathrm{F})$ of $5 \times 10^{6}$ GSES cells. Transgenic ES cells were grown in high-glucose Dulbecco's modified Eagle medium supplemented with $10 \%$ heat-inactivated ES cell-qualified fetal calf serum, $2 \mathrm{mM}$ L-glutamine, $50 \mathrm{U} / \mathrm{ml}$ penicillin, $50 \mu \mathrm{g} / \mathrm{ml}$ streptomycin, $1 \times$ nonessential amino acids, $0.4 \mathrm{mg} / \mathrm{ml}$ geneticin (G418; all reagents from Gibco BRL) and $0.1 \mathrm{~mm} \beta$-mercaptoethanol (Sigma). Antibiotic treatment was performed for 20 days before clonal expansion. Subsequently, stable lines were identified via genomic PCR using primers corresponding to the transfected plasmid. Primer 
Fig. 1. a DNA construct used for in vitro translation and stable expression of EGFP under control of the human 4.9-kb $\mathrm{Cx} 40$ promoter in Xenopus embryos and murine ES cells. b EGFP fluorescence in the abdominal aorta of a stage 45 tadpole previously injected with 100 pg Cx40-EGFP plasmid at the 2-cell stage. Scale bar corresponds to $200 \mu \mathrm{m}$. c Identification of stable Cx40-EGFP-transfected murine ES cell clones via genomic PCR using primers specific for the transfected plasmid. Black asterisks $=$ Positive clones revealing the expected band of 997 bp; white asterisk = positive control using the plasmid as template DNA.
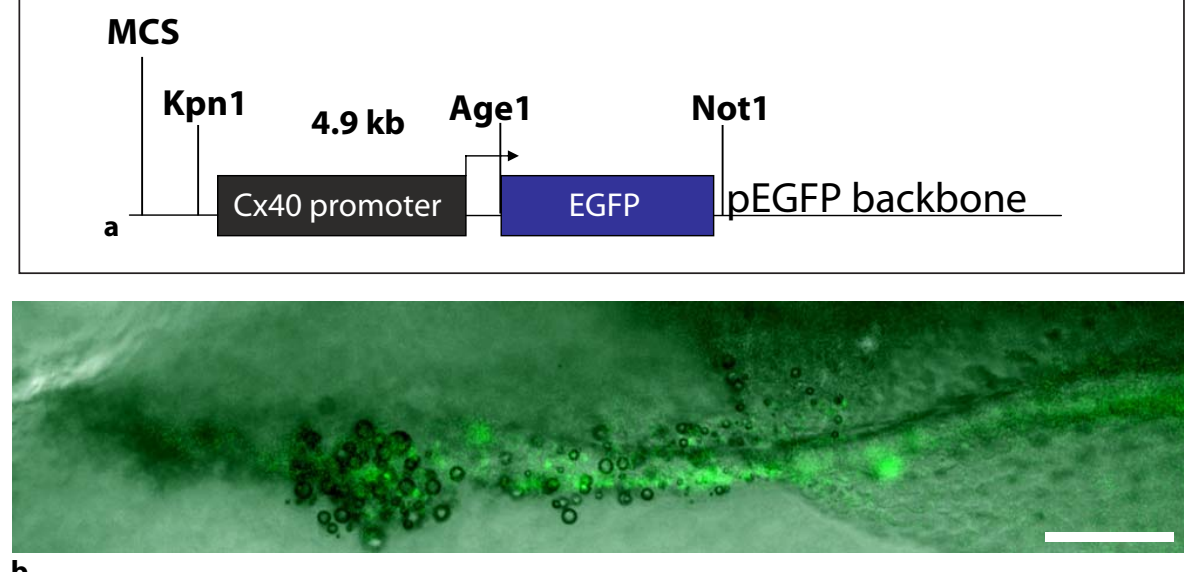

b

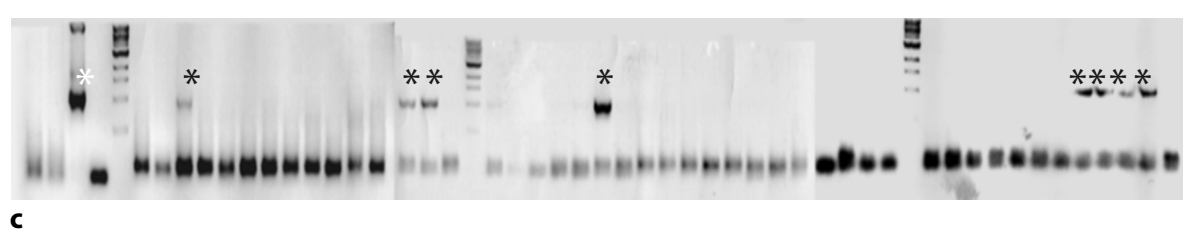

sequences were: 5'-GACAACCACTACCTGAGCAC-3' (forward primer) and 5'-CATTCCACAGCTGGTTCTTTCC-3' (backward primer). PCRs from positive clones yielded an amplified DNA fragment of the expected length (997 bp).

ES cell clones were kept undifferentiated under feeder-free conditions by addition of $1,000 \mathrm{U} / \mathrm{ml}$ purified recombinant mouse leukemia inhibitory factor (ESGRO; Life Technologies Inc.). Cells were maintained at $37^{\circ} \mathrm{C}$ in a humidified atmosphere of $5 \%$ $\mathrm{CO}_{2} / 95 \%$ air. Monolayers were passaged by trypsinization at confluence of $70-80 \%$. For FACS, differentiated cells were dissociated using PBS containing $5 \mathrm{mM}$ EDTA as described below. In vitro differentiation was initiated as follows: GSES cells were harvested with $0.25 \%$ trypsin-EDTA and dissociated cells were transferred to bacteriological dishes at a density of $2 \times 10^{5} \mathrm{ES}$ cells $/ \mathrm{ml}$ in Iscove's modified Eagle medium (Sigma) supplemented with $10 \%$ heat-inactivated fetal calf serum, $2 \mathrm{mM} \mathrm{L}$-glutamine, $50 \mathrm{U} / \mathrm{ml}$ penicillin, $50 \mu \mathrm{g} / \mathrm{ml}$ streptomycin, $1 \times$ nonessential amino acids (all reagents from Life Technologies Inc.) and $450 \mu \mathrm{M} \alpha$-monothioglycerol (Sigma). After 2 days, EBs were transferred to new medium. At day 6, EBs with a similar size were plated onto gelatin-coated tissue culture dishes. The growth medium for the attached differentiation cultures was changed every day.

Reverse Transcription Polymerase Chain Reaction

Semiquantitative RT-PCR incorporating $\alpha^{32} \mathrm{P}$-dCTP was performed according to standard protocols using RNA isolated from $5 \times 10^{4}$ FACS-purified or unpurified cells at day 6 of differentiation via the RNeasy Kit (Qiagen). The amplified murine cDNA fragments corresponded to bp 64-189 of $\mathrm{H} 4$, to bp 454-557 of Cx 40 , to bp 1332-1454 of Nkx2.5, to bp 5-260 of MLC2 $\mathrm{v}$ and to bp 65-270 of vascular endothelial cadherin. The annealing temperature was $57^{\circ} \mathrm{C}$ for all primer pairs, and the number of cycles used was between 28 and 33. The radioactive PCR products were separated on $7.5 \%$ polyacrylamide gels and analyzed using a phosphorimager.

\section{Flow Cytometry}

For FACS analysis of EGFP expression, the cells were dissociated in PBS containing $5 \mathrm{mM}$ EDTA for $15 \mathrm{~min}$ at $37^{\circ} \mathrm{C}$ after washing them twice in PBS without calcium. Subsequently, the cells were spinned down at 2,500 rpm for $3 \mathrm{~min}$ in an Eppendorf centrifuge and resuspended in $100 \mu \mathrm{l}$ ice-cold PBS containing $2 \%$ BSA. FACS analyses were performed with an Epics XL (BeckmanCoulter) using the evaluation program EXPO32ADC.

\section{Fluorescence Microscopy}

Adherent EBs were rinsed 3 times with PBS and fixed with 4\% paraformaldeyhde for $30 \mathrm{~min}$ at room temperature. The fixed cells were washed twice. Unspecific binding sites were blocked by PBS containing 20\% bovine serum and $0.05 \%$ Tween 20 for $1 \mathrm{~h}$. Primary antibodies anti-von Willebrand factor (Santa Cruz; dilution 1:100) and anti- $\alpha$-actinin (Sigma; dilution 1:250) were applied without washing and incubated for $30 \mathrm{~min}$ at $37^{\circ} \mathrm{C}$ with gentle shaking. After washing, cells were incubated with a Cy3conjugated goat anti-rabbit-IgG monoclonal antibody for $1 \mathrm{~h}$. All dilutions of antibodies were prepared in PBS containing 20\% goat serum. Controls of autofluorescence or nonspecific fluorescence were performed on fixed cells processed without the secondary and primary antibody. Monolayers were mounted in Mowiol and examined using a Zeiss Axiovert fluorescence microscope.

\section{Results}

We first isolated a $4.9-\mathrm{kb}$ fragment of the human $\mathrm{Cx} 40$ promoter and inserted it in pEGFP (Clontech) to enable cardiovascular-specific EGFP expression (fig. 1a). We subsequently used this construct for injections 


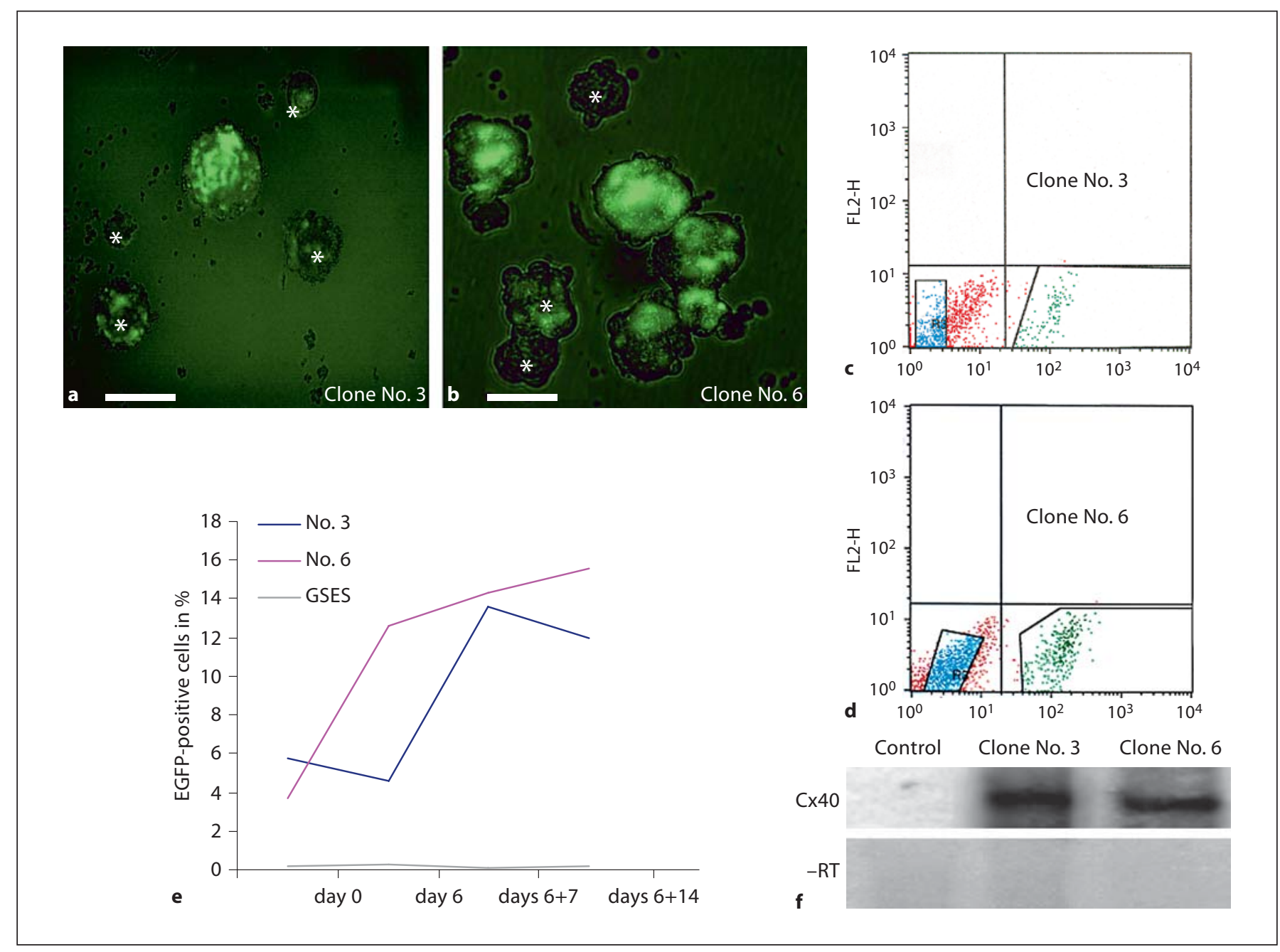

Fig. 2. Functionality of the Cx40-EGFP marker construct in stably transfected ES cells. a, b EGFP-positive EBs of 2 independent clones at day 6 of differentiation in suspension culture. Asterisks indicate EBs weakly expressing EGFP. Scale bars correspond to $100 \mu \mathrm{m}$. c, d FACS analysis of dissociated Cx40-EGFP-positive EBs derived from the same clones 13 days after differentiation, showing an EGFP-positive fraction of 8 and 17\%. e Time course of EGFP expression in the same 2 clones analyzed by FACS. GSES cells served as control. $f$ RT-PCR at day 6 of differentiation: strong enrichment of endogenous murine Cx40 mRNA after FACS purification of Cx40-EGFP-positive cells. GSES cells served as control. in Xenopus embryos, a convenient screening system routinely performed by our group to test for the functionality of promoter fragments or overexpressed factors [David et al., 2005]. We thereby found EGFP fluorescence specifically in the abdominal aorta of stage 45 tadpoles (fig. 1b), indicating a very high conservation of regulatory elements within the $4.9-\mathrm{kb}$ promoter fragment between higher and lower vertebrates. This encouraging observation prompted us to proceed with electroporations of murine ES cells. After subsequent clone selection using $0.4 \mathrm{mg} / \mathrm{ml}$ geneticin (G418) for 20 days, 8 stable lines were identified via genomic PCR with primers specific for the transfected plasmid (fig. 1c). The cells were induced to differentiate and analyzed for EGFP positivity via fluorescence microscopy after 6 days (fig. 2a, b). Typically, at that time point the EBs showed either large EGFP-positive areas or remained mainly EGFP negative (see asterisks in fig. 2a, b). FACS analyses at day 13 confirmed the visually obtained results and revealed positive fractions of $12-18 \%$ of cells within the EBs (fig. 2c, d). For further analysis, 2 of the clones were used in analytic FACS to define the time 

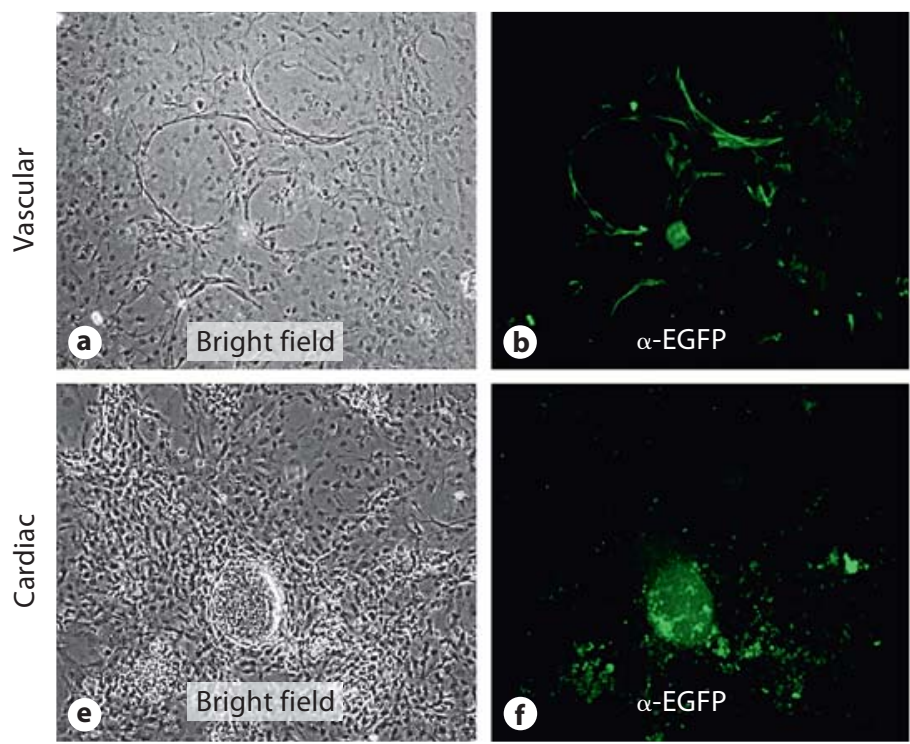

Fig. 3. Colocalization of EGFP-positive cells with von Willebrand factor (vWF) expression within vascular structures and $\alpha$-actinin expression within beating areas. a Vascular-like structures at day 13 of differentiation. b Immunofluorescent staining of EGFP. c Immunofluorescent staining of vWF. d Overlay of EGFP and
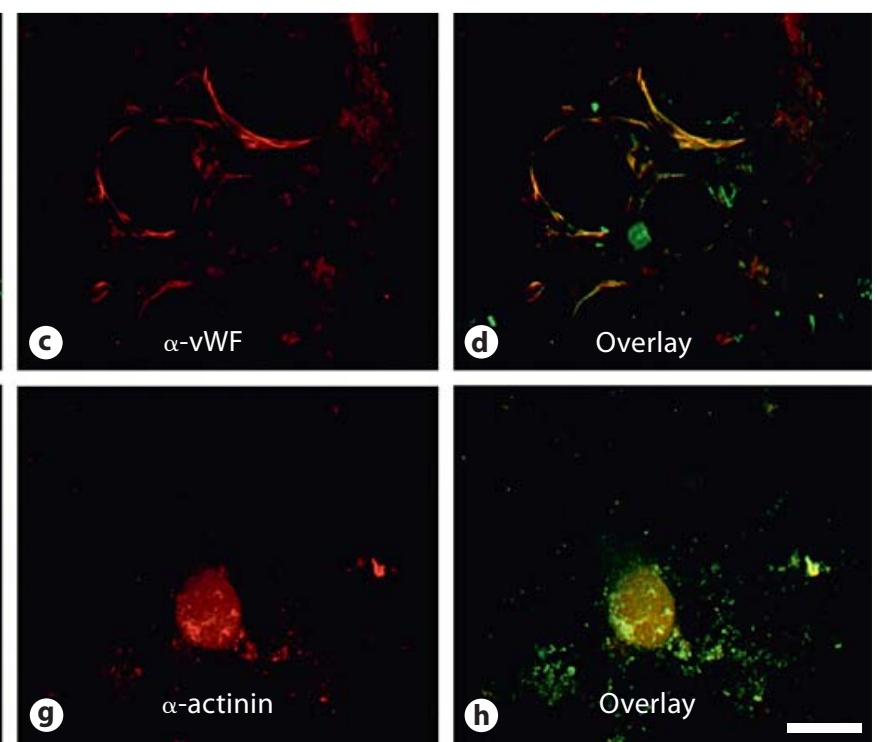

vWF stainings. e Beating area at day 13 of differentiation. $\mathbf{f}$ Immunofluorescent staining of EGFP. $\mathbf{g}$ Immunofluorescent staining of $\alpha$-actinin. $\mathbf{h}$ Overlay of EGFP and $\alpha$-actinin stainings. Scale bar corresponds to $20 \mu \mathrm{m}$.
Fig. 4. Enrichment of Cx40-EGFP-positive cells by manual isolation leads to enhanced cardiovasculogenesis. a Three-fold enrichment of Cx40-EGFP-positive cells derived from 6-day-old EBs verified via FACS. b Incidence of beating areas and vascular structures derived from unselected versus highly Cx40-EGFP-positive EBs. Each bar represents 24 independent wells, into each of which 5 EBs of equal size were plated at day 6 and analyzed at day 13 of differentiation.

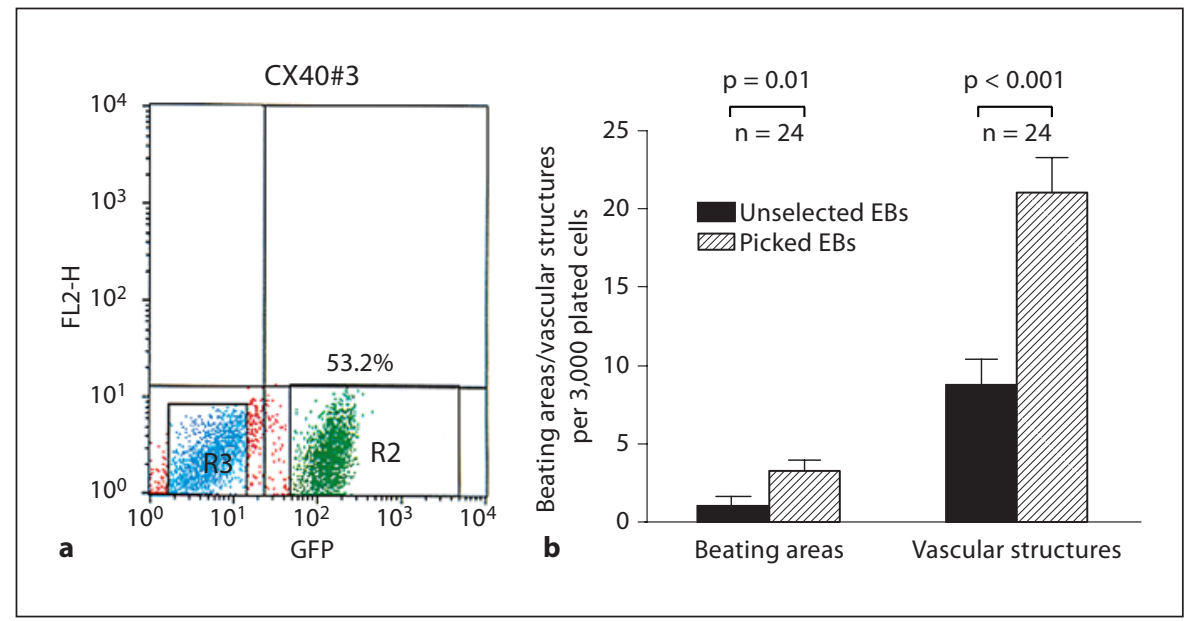

course of EGFP expression under control of the 4.9-kb $\mathrm{Cx} 40$ promoter fragment. We thereby found very weak expression at the onset of differentiation, yet a subsequent strong increase in $\mathrm{Cx} 40$-EGFP-positive cells up to day 20 when the maximal fluorescence was reached (fig. 2e). To verify the functionality of the $4.9-\mathrm{kb}$ human Cx40 promoter fragment in murine ES cells, we then performed preparative FACS followed by RT-PCRs for endogenous murine $\mathrm{Cx} 40 \mathrm{mRNA}$ expression which was dramatically enriched in the Cx40-EGFP-positive fractions (fig. 2f).

These results were confirmed on the protein level via immunofluorescent stainings showing EGFP expression exclusively in vascular-like structures simultaneously expressing von Willebrand factor (fig. 3a-d) and in formerly beating areas expressing $\alpha$-actinin (fig. $3 \mathrm{e}-\mathrm{h}$ ).

Based on these results, we next asked whether the 4.9$\mathrm{kb} \mathrm{Cx} 40$ promoter may be a suitable tool to isolate pure 


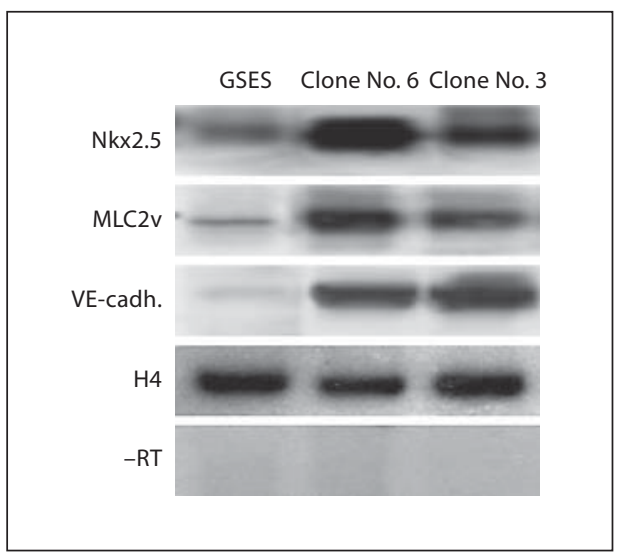

Fig. 5. Increased expression of cardiovascular markers in $\mathrm{Cx} 40$ EGFP-positive cells. Cells from 2 independent clones were sorted via FACS at day 6 of differentiation. mRNA expression of Nkx2.5, MLC2v and vascular endothelial cadherin (VE-cadh.) was determined by RT-PCR incorporating ${ }^{32} \mathrm{P}$. Unsorted ES cells served as control.

cardiovascular progenitor cells. We chose day 6 of differentiation at which time point $\mathrm{Cx} 40$-controlled EGFP expression had reached a submaximal level as described above (fig. 2e). To avoid FACS purification, which is harmful for further differentiation of ES cells when performed after differentiation day 3 [David et al., 2005], we chose a manual selection protocol based on EGFP fluorescence under the stereomicroscope. We thereby used a 20 - $\mu$ l pipette to select for highly EGFP-positive EBs in suspension culture such as shown for 1 EB in figure 2 a. Using this rather crude method we were able to enrich the EGFP-positive fraction approximately 3-fold (fig. 4a). Correspondingly, after further differentiation until day 13 , beating cardiomyocytes appeared with a 2 - to 3 -fold higher incidence than in unselected EBs and similar results were obtained for the appearance of vascular-like structures (fig. 4b). Beating frequencies ranged from 50 to $80 \mathrm{bpm}$ and did not differ between the selected and unselected EBs as well as untransfected controls (data not shown).

We next sought to verify these observations on the mRNA level with respect to specific marker expression. Thereby, semiquantitative RT-PCRs confirmed an enhanced cardiovascular differentiation after FACS purification based on Cx40-EGFP expression as shown for the cardiac markers $\mathrm{Nkx} 2.5$ and MLC2v as well as the endothelial marker vascular endothelial cadherin (fig. 5).

Cx40-Based Enrichment of ES Cell-

Derived Cardiovascular Progenitor Cells

\section{Discussion}

Cardiovascular diseases are the most frequent cause of death in the Western world. The critical loss of functional cardiomyocytes causes severe deterioration of pump function resulting in heart failure. As cardiomyocytes cannot be regenerated in adults, current therapeutic modalities for the treatment of end-stage heart failure are limited and include medical therapy, mechanical left ventricular assist devices and cardiac transplantation [Kessler and Byrne, 1999]. The latter is still the treatment of choice for end-stage heart failure. However, its application is limited by the availability of donor organs and the immune response, which requires life-long immunosuppressive therapy. Even in case of successful transplantation, frequent failure of donor organs mainly due to transplantation vasculopathy remains still unsolved [Hunt, 1998]. The possibility to regenerate or repair damaged or ischemic cardiac and vascular tissue is therefore a great challenge for the future.

So far, ES cells are the only cellular source that have been reproducibly shown to differentiate into cardiovascular tissue in vitro, making them a possible future tool to repair degenerative cardiovascular diseases [Balsam et al., 2004; Murry et al., 2004]. In many cases, however, such as cardiomyocytes, an appropriate endogenous marker is not known, and sorting methods have to rely on the introduction of a marker gene under the control of a lineage-specific promoter [David et al., 2005]. Therefore, transgenic purification strategies have to be developed to achieve high cell numbers for transplantation or cardiovascular tissue engineering. For the latter, the availability of cardiovascular precursor cells will be of particular interest. Importantly, cardiac and vascular cells share the same embryonic origin within the lateral plate mesoderm [Saga et al., 1999] and the heart is the first functional organ of the body and begins to pump soon after the vascular system of the embryo has established its first circulatory loops [Gilbert et al., 2000]. Rather than sprouting from the heart, blood vessels form independently, linking up to the heart soon afterwards [Larsen, 1998; Gilbert et al., 2000]. The rationale for our work was the observation that during development, $\mathrm{Cx} 40$ is endogenously expressed in cardiogenic cells as well as in the prospective ventricular conduction system [Teunissen and Bierhuizen, 2004]. In addition, vasculogenic as opposed to smooth muscle cells express $\mathrm{Cx} 40$ [Simon and McWhorter, 2002]. Whereas Cx40 expression during mammalian cardiovascular development has been well mapped by others [Delorme et al., 1997], our own results show a very high conservation of $\mathrm{Cx} 40$ promoter

Cells Tissues Organs 2008;188:62-69 
specificity even between mammalians and lower vertebrates. This is confirmed by the functionality of our human Cx40 promoter construct in the abdominal aorta of frog tadpoles after reporter plasmid injection (fig. 1b).

This high conservation of $\mathrm{Cx} 40$ promoter regulation is also reflected by a significant enrichment of functional ES cell-derived cardiomyocytes accompanied by a simultaneous increase in vascular cells after transference of our novel $\mathrm{Cx} 40$ promoter-based approach to ES cells. This may become very important for future ES cell-based cardiac cell therapy.

Further, our ES cell-based data reveal that after crude manual enrichment of highly Cx40-EGFP-positive EBs, which led to an approximately 3 -fold enrichment of the exact number of Cx40-EGFP-positive cells, the appearance of cardiac and vascular structures was increased by the same factor. Again, this shows the high specificity of our 4.9-kb promoter construct as observed on the levels of phenotypic analysis as well as in extensive RT-PCR analyses and protein expression data (fig. 2d, 3, 4).

Our study aimed to investigate the feasibility of a future high-grade purification of cardiovasculogenic cells from differentiating EBs using the $\mathrm{Cx} 40$ promoter. It shows proof of principle that the deleted CD4 $(\Delta \mathrm{CD} 4)$ surface marker-based method for MACS previously described by us appears well suitable for transference to this promoter [David et al., 2005]. Potential limitations of this approach are contaminations with undifferentiated cells, which may be tumorigenic, or with undesired cell types eventually leading to arrhythmias. On the other hand, promoters displaying a very weak activity may lead to levels of $\triangle C D 4$ expression too low for an efficient purification. However, MACS is currently regarded as the gold standard for mild and time-sparing cell purification. Using MACS, up to $10^{11}$ cells can be analyzed in about $1 \mathrm{~h}$, making it possible to separate large cell numbers and identify even rare populations of cells.

We have recently developed this novel technique to overcome the obstacles associated with alternative approaches relying on cytometry, which is slow and typically capable of analyzing no more than 3,000 cells/s. To achieve cell numbers required for transplantations into humans ( $>10^{8}$ cells in the case of cardiomyocytes), a purification period of more than $500 \mathrm{~h}$ would be necessary. Therefore, FACS does not appear to provide the capabilities to identify a rare population of cells or to separate large numbers of cells due to the excessive amount of analysis and sorting time [David et al., 2005]. Likewise, approaches relying on the introduction of drug resistance genes for antibiotic selection [Klug et al., 1996; Zandstra et al., 2003] are critical because of the long incubation period with the hazard of resistance and possible harmful effects of the antibiotic on terminally differentiated cells themselves. Additionally, FACS sorting based on fluorescent markers as well as antibiotic selection are based on the expression of nonhuman proteins that may cause additional immunological problems or even be toxic in patients.

Due to our encouraging data and the urgent need of cardiovasculogenic cell material as a basis for tissue engineering and cell transplantation studies, we will now combine the $\triangle \mathrm{CD} 4$-based MACS technique with the Cx40 promoter approach. Thereby, we will generate stable human ES cell lines bearing the $\mathrm{Cx} 40-\Delta \mathrm{CD} 4$ promoter construct in order to dispose of human cardiovasculogenic cell material valuable in preclinical studies using immunodeficient mice as recipients. Likewise, the $\mathrm{Cx} 40$ positive cells selected via this gentle MACS-based method will be used for detailed functional characterization with respect to their pharmacological and electrophysiological properties. These are likely to be affected in the FACS-sorted cells used in our initial work reported here. Furthermore, it will be of great interest to transfer the Cx40- $\Delta$ CD4-based approach to the recently described spermatogonial stem cells [Guan et al., 2006] as well as to stem cells derived from reprogrammed somatic cells [Takahashi and Yamanaka, 2006; Park and Daley, 2007; Takahashi et al., 2007; Wernig et al., 2007]. This may be the basis for future autologous cellular sources and future clinical use of $\mathrm{Cx} 40$ promoter-purified cardiovascular progenitor cells.

\section{Acknowledgements}

We are very grateful to Christiane Gross who is funded by the DFG (FR 705/11-3) and the Fritz-Bender-Stiftung and to Judith Arcifa for expert technical assistance. R.D. is funded exclusively by the DFG (FR 705/11-3). Additional funding was granted by the Dr. Helmut Legerlotz-Stiftung. We also wish to thank Prof. Ralph Rupp for providing the Xenopus facilities.

References

Balsam, L.B., A.J. Wagers, J.L. Christensen, T. Kofidis, I.L. Weissman, R.C. Robbins (2004) Haematopoietic stem cells adopt mature haematopoietic fates in ischaemic myocardium. Nature 428: 668-673.

David, R., T.O. Joos, C. Dreyer (1998) Anteroposterior patterning and organogenesis of Xenopus laevis require a correct dose of germ cell nuclear factor (xGCNF). Mech Dev 79: $137-152$. 
David, R., M. Groebner, W.M. Franz (2005) Magnetic cell sorting purification of differentiated embryonic stem cells stably expressing truncated human CD4 as surface marker. Stem Cells 23: 477-482.

Delorme, B., E. Dahl, T. Jarry-Guichard, J.P. Briand, K. Willecke, D. Gros, M. Théveniau-Ruissy (1997) Expression pattern of connexin gene products at the early developmental stages of the mouse cardiovascular system. Circ Res 81: 423-437.

Gerecht-Nir, S., B. Fishman, J. Itskovitz-Eldor (2004) Cardiovascular potential of embryonic stem cells. Anat Rec A Discov Mol Cell Evol Biol 276: 58-65.

Gilbert, S.F., M.S. Tyler, R.N. Kozlowski (2000) Developmental Biology, ed 6. Sunderland, Sinauer Associates.

Guan, K., K. Nayernia, L.S. Maier, S. Wagner, R. Dressel, J.H. Lee, J. Nolte, F. Wolf, M. Li, W. Engel, G. Hasenfuss (2006) Pluripotency of spermatogonial stem cells from adult mouse testis. Nature 440: 1199-1203.

Hunt, S.A. (1998) Current status of cardiac transplantation. JAMA 280: 1692-1698.

Kessler, P.D., B.J. Byrne (1999) Myoblast cell grafting into heart muscle: cellular biology and potential applications. Annu Rev Physiol 61: 219-242.

Klug, M.G., M.H. Soonpaa, G.Y. Koh, L.J. Field (1996) Genetically selected cardiomyocytes from differentiating embronic stem cells form stable intracardiac grafts. J Clin Invest 98: 216-224.

Larsen, W.J. (1998) Essentials of Human Embryology, ed 2. New York, Churchill Livingstone.
Li, R.K., Z.Q. Jia, R.D. Weisel, F. Merante, D.A Mickle (1999) Smooth muscle cell transplantation into myocardial scar tissue improves heart function. J Mol Cell Cardiol 31: $513-$ 522.

Muller, M., B.K. Fleischmann, S. Selbert, G.J. Ji, E. Endl, G. Middeler, O.J. Muller, P. Schlenke, S. Frese, A.M. Wobus, J. Hescheler, H.A. Katus, W.M. Franz (2000) Selection of ventricular-like cardiomyocytes from ES cells in vitro. FASEB J 14: 2540-2548.

Murry, C.E., M.H. Soonpaa, H. Reinecke, H. Nakajima, H.O. Nakajima, M. Rubart, K.B. Pasumarthi, J.I. Virag, S.H. Bartelmez, V. Poppa, G. Bradford, J.D. Dowell, D.A. Williams, L.J. Field (2004) Haematopoietic stem cells do not transdifferentiate into cardiac myocytes in myocardial infarcts. Nature 428 : 664-668.

Nir, S.G., R. David, M. Zaruba, W.M. Franz, J. Itskovitz-Eldor (2003) Human embryonic stem cells for cardiovascular repair. Cardiovasc Res 58: 313-323.

Oyamada, Y., K. Komatsu, H. Kimura, M. Mori, M. Oyamada (1996) Differential regulation of gap junction protein (connexin) genes during cardiomyocytic differentiation of mouse embryonic stem cells in vitro. Exp Cell Res 229: 318-326.

Park, I.H., G.Q. Daley (2007) Debugging cellular reprogramming. Nat Cell Biol 9: 871-873.

Saga, Y., S. Miyagawa-Tomita, A. Takagi, S. Kitajima, J. Miyazaki, T. Inoue (1999) MesP1 is expressed in the heart precursor cells and required for the formation of a single heart tube. Development 126: 3437-3447.
Seul, K.H., P.N. Tadros, E.C. Beyer (1997) Mouse connexin40: gene structure and promoter analysis. Genomics 46: 120-126.

Simon, A.M., A.R. McWhorter (2002) Vascular abnormalities in mice lacking the endothelial gap junction proteins connexin37 and connexin40. Dev Biol 251: 206-220.

Takahashi, K., S. Yamanaka (2006) Induction of pluripotent stem cells from mouse embryonic and adult fibroblast cultures by defined factors. Cell 126: 663-676.

Takahashi, K., K. Tanabe, M. Ohnuki, M. Narita, T. Ichisaka, K. Tomoda, S. Yamanaka (2007) Induction of pluripotent stem cells from adult human fibroblasts by defined factors. Cell 131: 861-872.

Teunissen, B.E., M.F. Bierhuizen (2004) Transcriptional control of myocardial connexins. Cardiovasc Res 62: 246-255

Vittet, D., M.H. Prandini, R. Berthier, A. Schweitzer, H. Martin-Sisteron, G. Uzan, E. Dejana (1996) Embryonic stem cells differentiate in vitro to endothelial cells through successive maturation steps. Blood 88: 34243431.

Wernig, M., A. Meissner, R. Foreman, T. Brambrink, M. Ku, K. Hochedlinger, B.E. Bernstein, R. Jaenisch (2007) In vitro reprogramming of fibroblasts into a pluripotent ES-cell-like state. Nature 448: 318-324.

Zandstra, P.W., C. Bauwens, T. Yin, Q. Liu, H. Schiller, R. Zweigerdt, K.B. Pasumarthi, L.J. Field (2003) Scalable production of embryonic stem cell-derived cardiomyocytes. Tissue Eng 9: 767-778. 